\title{
Estimation-based Norm-optimal Iterative Learning Control
}

\author{
Patrik Axelsson, Rickard Karlsson, Mikael Norrlöf \\ Division of Automatic Control \\ E-mail: axelsson@isy.liu.se, rickard@isy.liu.se, \\ mino@isy.liu.se
}

18th October 2013

Report no.: LiTH-ISY-R-3066

Submitted to Automatica

Address:

Department of Electrical Engineering

Linköpings universitet

SE-581 83 Linköping, Sweden

WWW: http://wwW.control.isy.liu.se

AUTOMATIC CONTROL

REGLERTEKNIK

LINKÖPINGS UNIVERSITET

Technical reports from the Automatic Control group in Linköping are available from http://www. control.isy.liu.se/publications. 


\begin{abstract}
The iterative learning control (ILC) method improves performance of systems that repeat the same task several times. In this paper the standard norm-optimal ILC control law for linear systems is extended to an estimation-based ILC algorithm where the controlled variables are not directly available as measurements. The proposed ILC algorithm is proven to be stable and gives monotonic convergence of the error. The estimationbased part of the algorithm uses Bayesian estimation techniques such as the Kalman filter. The objective function in the optimisation problem is modified to incorporate not only the mean value of the estimated variable, but also information about the uncertainty of the estimate. It is further shown that for linear time-invariant systems the ILC design is independent of the estimation method. Finally, the concept is extended to non-linear state space models using linearisation techniques, where it is assumed that the full state vector is estimated and used in the ILC algorithm. It is also discussed how the Kullback-Leibler divergence can be used if linearisation cannot be performed. Finally, the proposed solution for non-linear systems is applied and verified in a simulation study with a simplified model of an industrial manipulator system.
\end{abstract}

Keywords: Iterative, Learning Control, Estimation, Filtering, Nonlinear systems, Optimal 


\title{
Estimation-based Norm-optimal Iterative Learning Control ${ }^{\star}$
}

\author{
Patrik Axelsson ${ }^{\text {a }}$, Rickard Karlsson ${ }^{\text {b }}$, Mikael Norrlöf ${ }^{\text {a }}$ \\ ${ }^{a}$ Division of Automatic Control, Department of Electrical Engineering, Linköping University, SE-581 83 Linköping, Sweden \\ b Nira Dynamics, Teknikringen 6, SE-583 30 Linköping, Sweden
}

\begin{abstract}
The iterative learning control (ILC) method improves performance of systems that repeat the same task several times. In this paper the standard norm-optimal ILC control law for linear systems is extended to an estimation-based ILC algorithm where the controlled variables are not directly available as measurements. The proposed ILC algorithm is proven to be stable and gives monotonic convergence of the error. The estimation-based part of the algorithm uses Bayesian estimation techniques such as the Kalman filter. The objective function in the optimisation problem is modified to incorporate not only the mean value of the estimated variable, but also information about the uncertainty of the estimate. It is further shown that for linear time-invariant systems the ILC design is independent of the estimation method. Finally, the concept is extended to non-linear state space models using linearisation techniques, where it is assumed that the full state vector is estimated and used in the ILC algorithm. It is also discussed how the Kullback-Leibler divergence can be used if linearisation cannot be performed. Finally, the proposed solution for non-linear systems is applied and verified in a simulation study with a simplified model of an industrial manipulator system.
\end{abstract}

Key words: Iterative, Learning Control, Estimation, Filtering, Nonlinear systems, Optimal

\section{Introduction}

The iterative learning control (ILC) method, [5,21], improves performance, for instance trajectory tracking accuracy, for systems that repeat the same task several times. Traditionally, a successful solution to many such problems can base the ILC control law on direct measurements of the control quantity. When this quantity is not directly available as a measurement, it has to be estimated from other measurements, [27]. In this paper the estimation-based ILC framework from [27] is combined with an ILC design based on an optimisation approach, referred to as norm-optimal ILC $[2,3]$. In addition, the estimation problem is formulated using recursive Bayesian methods, where the Kalman filter (KF) can be applied for linear systems. Extensions to nonlinear systems are possible using linearisation, extended Kalman filter (EKF), or simulation based methods such as the particle filter (PF), [12,13]. Using batch formulation of data it is possible to analyse stability and station-

\footnotetext{
^ This paper was not presented at any IFAC meeting. Corresponding author. P. Axelsson. Tel. +4613281365.

Email addresses: patrik.axelsson@liu.se (Patrik Axelsson), rickard.karlsson@niradynamics.se (Rickard Karlsson), mikael.norrlof@liu.se (Mikael Norrlöf).
}

ary performance of the ILC algorithm. Depending on the system assumption and estimator choice it is also possible to base the derivation of the optimal control law on different optimization criteria.

Previous work on ILC in combination with KF can be found in e.g. $[1,19]$. In $[1,19]$ it is assumed that the controlled variable is the measured variable, affected by measurement noise. A model of the control error as a function of the ILC iteration index is developed and a $\mathrm{KF}$ is applied in order to reduce the measurement noise. The design of the ILC update law in [19] uses a quadratic criterion optimisation-based ILC and in [1] a P-ILC algorithm is used. In [25-27] the measured and controlled variable does not coincide. Therefore, estimation techniques are used to get the unknown state variables which are used in the ILC algorithm. In [26], the performance for a two-link flexible industrial robot is improved using filter-based ILC and an EKF to estimate the arm angles. Estimation-based ILC is also used in [25] applied to a Gantry-Tau parallel kinematic robot where a KF and a complementary filter is used for estimation. Theoretical properties of the estimation-based ILC are investigated in [27]. Estimation-based ILC using more advanced Bayesian techniques than EKF such as PF and unscented Kalman filter have been used in [8] together with filterbased ILC. This paper generalizes the ideas in [8,25-27] 
to the case where the full probability density function (PDF) of the estimated quantity is used in an optimisation problem similar to the norm-optimal ILC $[2,3]$. In addition, the full knowledge of the estimated state vector from previous ILC iteration, enables non-linear extensions by utilising linearisation solutions in the proposed estimation-based ILC method.

This paper is organised as follows. In Section 2 an overview of ILC is given, where the norm-optimal ILC method is treated in detail. Section 3 discusses Bayesian filtering techniques. In Section 4 estimation-based ILC is defined, and extensions of the objective function are presented as well as stability and convergence results. Section 5 extends previous results to non-linear systems. Finally, Section 6 shows some simulation results.

\section{Iterative Learning Control}

The iterative learning control (ILC) method improves the performance of systems that repeat the same task multiple times. The ILC control signal $\mathbf{u}_{k+1}(t) \in \mathbb{R}^{n_{u}}$ for the next iteration is calculated using the error signal and the ILC control signal, both from the current iteration $k$. A common update law is given by

$$
\mathbf{u}_{k+1}(t)=\mathcal{Q}(q)\left(\mathbf{u}_{k}(t)+\mathcal{L}(q) \mathbf{e}_{k}(t)\right)
$$

where $\mathbf{e}_{k}(t)$ is the ILC error, $q$ is the time-shift operator, $t$ is time and $k$ is the ILC iteration index. The filters $\mathcal{Q}(q)$ and $\mathcal{L}(q)$ are possibly non-causal. In the design of the filters, there is a trade off between convergence speed, error reduction and plant knowledge used in the design process. If the filter $\mathcal{Q}(q)$ is omitted and the filter $\mathcal{L}(q)$ is chosen as the inverse of the system, then the error converges to zero in one iteration. However, inverting the system will be very sensitive to model errors and may result in a very non-robust solution. Instead, the choice $\mathcal{L}(q)=\gamma q^{j}$ is often used, where $0<\gamma<1$ and $j$ a positive integer are the design variables. Moreover, the filter $\mathcal{Q}(q)$ is introduced in order to restrict the high frequency influence from the error and also reduce the influence of measurement noise. Including $\mathcal{Q}(q)$ makes the ILC algorithm converging slower and to a non zero error. More on system related properties for ILC, see $[11,23]$. Other choices of ILC updating laws can be found in $[10,11,21]$, where the norm-optimal ILC algorithm is the one used in this paper.

\subsection{Norm-optimal ILC}

In this section, the norm-optimal ILC algorithm will be explained for the common case where the measured variables $\mathbf{y}_{k}(t)$ and the controlled variables $\mathbf{z}_{k}(t) \in \mathbb{R}^{n_{z}}$ coincide. A thorough description of the norm-optimal ILC al- gorithm can be found in e.g. $[2,3,14]$. The method solves

$$
\begin{array}{ll}
\underset{\mathbf{u}_{k+1}(\cdot)}{\operatorname{minimise}} & \sum_{t=0}^{N-1}\left\|\mathbf{e}_{k+1}(t)\right\|_{\mathbf{W}_{\mathbf{e}}}^{2}+\left\|\mathbf{u}_{k+1}(t)\right\|_{\mathbf{W}_{\mathbf{u}}}^{2} \\
\text { subject to } & \left\|\mathbf{u}_{k+1}(t)-\mathbf{u}_{k}(t)\right\|^{2} \leq \delta
\end{array}
$$

where $\mathbf{e}_{k+1}(t)=\mathbf{r}(t)-\mathbf{z}_{k+1}(t)$ is the error, $\mathbf{W}_{e} \in \mathbb{S}_{++}^{n_{z}}$, and $\mathbf{W}_{u} \in \mathbb{S}_{++}^{n_{u}}$ are weight matrices for the error and the ILC control signal, respectively ${ }^{1}$. Let the Lagrangian function [22] be defined by

$$
\begin{aligned}
\mathfrak{L}\left(\mathbf{u}_{k+1}(t), \lambda\right) \triangleq & \frac{1}{2} \sum_{t=0}^{N-1}\left\|\mathbf{e}_{k+1}(t)\right\|_{\mathbf{W}_{e}}^{2}+\left\|\mathbf{u}_{k+1}(t)\right\|_{\mathbf{W}_{u}}^{2} \\
& +\lambda\left\|\mathbf{u}_{k+1}(t)-\mathbf{u}_{k}(t)\right\|^{2}-\lambda \delta
\end{aligned}
$$

where $\lambda \in \mathbb{R}_{+}$is the Lagrange multiplier. The firstorder sufficient conditions for optimality are given by the Karush-Kuhn-Tucker (KKT) conditions [22]. Instead of finding an optimal value of $\lambda$, it is assumed that $\lambda$ is a tuning parameter. By doing so, the optimum is obtained where the gradient of the Lagrangian function with respect to $\mathbf{u}_{k+1}(t)$ equals zero. The KKT conditions implies that the constraint $\left\|\mathbf{u}_{k+1}(t)-\mathbf{u}_{k}(t)\right\|^{2} \leq \delta$ will always be satisfied with equality and where the value of $\delta$ depends indirect of the value of $\lambda$. A large $\lambda$ implies a small $\delta$ and vice versa. The second-order sufficient condition for optimality is that the Hessian of the Lagrangian function with respect to $\mathbf{u}_{k+1}(t)$ is greater than zero.

Stacking the vectors $\mathbf{u}_{k}(t), \mathbf{z}_{k}(t)$, and $\mathbf{r}(t)$ for time $t=$ $0, \ldots, N-1$ at the same ILC iteration $k$ according to

$$
\begin{aligned}
& \mathbf{u}_{k}=\left(\mathbf{u}_{k}(0)^{\top} \ldots \mathbf{u}_{k}(N-1)^{\top}\right)^{\top} \in \mathbb{R}^{N n_{u}}, \\
& \mathbf{z}_{k}=\left(\mathbf{z}_{k}(0)^{\top} \ldots \mathbf{z}_{k}(N-1)^{\top}\right)^{\top} \in \mathbb{R}^{N n_{z}}, \\
& \mathbf{r}=\left(\mathbf{r}(0)^{\top} \ldots \mathbf{r}(N-1)^{\top}\right)^{\top} \in \mathbb{R}^{N n_{z}}
\end{aligned}
$$

which are known as super-vectors, gives

$$
\begin{aligned}
\mathfrak{L}=\frac{1}{2}\left[\mathbf{e}_{k+1}^{\top} \mathcal{W}_{\mathbf{e}} \mathbf{e}_{k+1}+\mathbf{u}_{k+1}^{\top} \mathcal{W}_{\mathbf{u}} \mathbf{u}_{k+1}\right. \\
\left.+\lambda\left(\mathbf{u}_{k+1}-\mathbf{u}_{k}\right)^{\top}\left(\mathbf{u}_{k+1}-\mathbf{u}_{k}\right)\right]
\end{aligned}
$$

where $\mathbf{e}_{k+1}=\mathbf{r}-\mathbf{z}_{k+1}$ and

$$
\begin{aligned}
& \mathcal{W}_{\mathbf{e}}=\mathbf{I}_{N} \otimes \mathbf{W}_{e} \in \mathbb{S}_{++}^{N n_{z}}, \\
& \mathcal{W}_{\mathbf{u}}=\mathbf{I}_{N} \otimes \mathbf{W}_{u} \in \mathbb{S}_{++}^{N n_{u}} .
\end{aligned}
$$

Here, $\mathbf{I}_{N}$ is the $N \times N$ identity matrix and $\otimes$ denotes the Kronecker product. In (5), the term including $\lambda \delta$ is omitted since it does not depend on $\mathbf{u}_{k+1}$.

\footnotetext{
$\overline{1} \mathbb{S}_{++}^{n}$ denotes a $n \times n$ positive definite matrix.
} 
Using the state space model

$$
\begin{aligned}
\mathbf{x}_{k}(t+1) & =\mathbf{A}(t) \mathbf{x}_{k}(t)+\mathbf{B}_{\mathbf{u}}(t) \mathbf{u}_{k}(t)+\mathbf{B}_{\mathbf{r}}(t) \mathbf{r}(t) \\
\mathbf{z}_{k}(t) & =\mathbf{C}_{\mathbf{z}}(t) \mathbf{x}_{k}(t)+\mathbf{D}_{\mathbf{z u}}(t) \mathbf{u}_{k}(t)+\mathbf{D}_{\mathbf{z r}}(t) \mathbf{r}(t),
\end{aligned}
$$

the batch description ${ }^{2}$ for $\mathbf{z}_{k}$ can be written as

$$
\mathbf{z}_{k}=\mathcal{C}_{\mathbf{z}} \boldsymbol{\Phi} \mathbf{x}_{0}+\mathbf{T}_{\mathbf{z u}} \mathbf{u}_{k}+\mathbf{T}_{\mathbf{z r}} \mathbf{r}
$$

where the details are given in Appendix A. The objective function (5) using $\mathbf{e}_{k+1}=\mathbf{r}-\mathbf{z}_{k+1}$ becomes

$$
\begin{aligned}
\mathfrak{L}= & \frac{1}{2} \mathbf{u}_{k+1}^{\top}\left(\mathbf{T}_{\mathbf{z u}}^{\top} \mathcal{W}_{\mathbf{e}} \mathbf{T}_{\mathbf{z u}}+\mathcal{W}_{\mathbf{u}}+\lambda \mathbf{I}\right) \mathbf{u}_{k+1} \\
& -\left(\left(\left(\mathbf{I}-\mathbf{T}_{\mathbf{z r}}\right) \mathbf{r}-\mathcal{C}_{\mathbf{z}} \mathbf{\Phi} \mathbf{x}_{0}\right)^{\top} \mathcal{W}_{\mathbf{e}} \mathbf{T}_{\mathbf{z u}}+\lambda \mathbf{u}_{k}^{\top}\right) \mathbf{u}_{k+1}
\end{aligned}
$$

where all terms independent of $\mathbf{u}_{k+1}$ are omitted.

As mentioned before, the minimum is obtained when the gradient of $\mathfrak{L}$ equals zero. The second-order sufficient condition is fulfilled, since the Hessian matrix $\mathbf{T}_{\mathbf{z u}}^{\top} \mathcal{W}_{\mathbf{e}} \mathbf{T}_{\mathbf{z u}}+\mathcal{W}_{\mathbf{u}}+\lambda \mathbf{I}$ is positive definite when $\mathcal{W}_{\mathbf{e}} \in$ $\mathbb{S}_{++}, \mathcal{W}_{\mathbf{u}} \in \mathbb{S}_{++}$, and $\lambda \in \mathbb{R}_{+}$. By solving the gradient equal to zero with respect to $\mathbf{u}_{k+1}$, and using (7) together with $\mathbf{e}_{k}=\mathbf{r}-\mathbf{z}_{k}$ to eliminate the terms involving $\mathbf{r}$ and $\mathbf{x}_{0}$ gives

$$
\begin{aligned}
\mathbf{u}_{k+1}= & \mathcal{Q} \cdot\left(\mathbf{u}_{k}+\mathcal{L} \cdot \mathbf{e}_{k}\right) \\
\mathcal{Q}= & \left(\mathbf{T}_{\mathbf{z u}}^{\top} \mathcal{W}_{\mathbf{e}} \mathbf{T}_{\mathbf{z u}}+\mathcal{W}_{\mathbf{u}}+\lambda \mathbf{I}\right)^{-1} \\
& \times\left(\lambda \mathbf{I}+\mathbf{T}_{\mathbf{z u}}^{\top} \mathcal{W}_{\mathbf{e}} \mathbf{T}_{\mathbf{z u}}\right) \\
\mathcal{L}= & \left(\lambda \mathbf{I}+\mathbf{T}_{\mathbf{z u}}^{\top} \mathcal{W}_{\mathbf{e}} \mathbf{T}_{\mathbf{z u}}\right)^{-1} \mathbf{T}_{\mathbf{z u}}^{\top} \mathcal{W}_{\mathbf{e}}
\end{aligned}
$$

where the matrices $\mathcal{Q}$ and $\mathcal{L}$ are not to be confused with the filters $\mathcal{Q}(q)$ and $\mathcal{L}(q)$ in $(1)$.

\subsection{Stability Analysis}

The stability can be analysed by considering the system in (7) with the ILC update law (8a). Using $\mathbf{e}_{k}=\mathbf{r}-\mathbf{z}_{k}$ gives the following iterative linear system

$$
\begin{aligned}
\mathbf{u}_{k+1}= & \mathcal{Q} \cdot\left(\mathbf{I}-\mathcal{L} \cdot \mathbf{T}_{\mathbf{z u}}\right) \mathbf{u}_{k} \\
& +\mathcal{Q} \cdot \mathcal{L} \cdot\left(\left(\mathbf{I}-\mathbf{T}_{\mathbf{z r}}\right) \mathbf{r}-\mathcal{C}_{\mathbf{z}} \mathbf{\Phi} \mathbf{x}_{0}\right)
\end{aligned}
$$

The linear iterative system can be treated as a normal discrete-time state space model when investigating stability. The stability requirement becomes $\rho\left(\mathcal{Q} \cdot\left(\mathbf{I}-\mathcal{L} \cdot \mathbf{T}_{\mathbf{z u}}\right)\right)<1$, where $\rho(\cdot)$ is the spectral radius, i.e., the maximal absolute value of the eigenvalues, see e.g. [24]. In [23, Theorem 9] a requirement for monotone convergence is presented, which is stronger than only stability. Stability and convergence properties for the norm-optimal ILC algorithm in (8) is given in Theorem 1.

\footnotetext{
${ }^{2}$ Also known as lifted system representation.
}

Theorem 1 (Stability and monotonic convergence): The iterative system (9) is stable and monotonically convergent for all system descriptions in (7) using $\mathcal{Q}$ and $\mathcal{L}$ from $(8)$.

PROOF. From [23, Theorem 9] it holds that the iterative system (9) is stable and converges monotonically if $\bar{\sigma}\left(\mathcal{Q} \cdot\left(\mathbf{I}-\mathcal{L} \cdot \mathbf{T}_{\text {zu }}\right)\right)<1$, where $\bar{\sigma}(\cdot)$ is the largest singular value. Using $\mathcal{Q}$ and $\mathcal{L}$ from (8) gives

$$
\mathcal{Q} \cdot\left(\mathbf{I}-\mathcal{L} \cdot \mathbf{T}_{\mathbf{z u}}\right)=\left(\mathbf{T}_{\mathbf{z u}}^{\top} \mathcal{W}_{\mathbf{e}} \mathbf{T}_{\mathbf{z u}}+\mathcal{W}_{\mathbf{u}}+\lambda \mathbf{I}\right)^{-1} \lambda .
$$

Since $\mathcal{W}_{\mathbf{e}} \in \mathbb{S}_{++}, \mathcal{W}_{\mathbf{u}} \in \mathbb{S}_{++}$, and $\lambda \in \mathbb{R}_{+}$it holds that the maximal singular value is less than one independent of the system description $\mathbf{T}_{\mathbf{z u}}$.

Note that the requirement $\bar{\sigma}\left(\mathcal{Q} \cdot\left(\mathbf{I}-\mathcal{L} \cdot \mathbf{T}_{\mathbf{z u}}\right)\right)<1$ is general and can be used with any matrices $\mathcal{Q}$ and $\mathcal{L}$ to prove stability and monotone convergence.

In [9] it is concluded that the smallest error is achieved if $\mathcal{W}_{\mathbf{u}}=0$, however $\mathcal{W}_{\mathbf{u}} \neq 0$ is used in order to handle model errors. Moreover, it is discussed that the convergence rate mostly depends on $\lambda$, where $\lambda=0$ gives deadbeat control w.r.t the iterations and $\lambda \rightarrow \infty$ makes the convergence rate arbitrarily slow.

Since the iterative system (9) is stable and monotonically convergent, it will converge to the asymptotic control signal $\mathbf{u}_{\infty}$ giving the asymptotic error $\mathbf{e}_{\infty}$. The stationary $\mathbf{u}_{\infty}$ and $\mathbf{e}_{\infty}$ can be obtained by using $\mathbf{u}_{k}=\mathbf{u}_{k+1}=\mathbf{u}_{\infty}$ and $\mathbf{e}_{\infty}=\mathbf{r}-\mathbf{z}_{\infty}$, see e.g. [9].

\section{Bayesian Estimation}

Consider the discrete-time state space model

$$
\begin{aligned}
\mathbf{x}(t+1) & =f(\mathbf{x}(t), \boldsymbol{\tau}(t), \mathbf{w}(t)) \\
\mathbf{y}(t) & =h(\mathbf{x}(t))+\mathbf{v}(t),
\end{aligned}
$$

with state variables $\mathbf{x}(t) \in \mathbb{R}^{n_{x}}$, input signal $\boldsymbol{\tau}(t) \in \mathbb{R}^{n_{\tau}}$ and measurements $\mathbf{y}(t) \in \mathbb{R}^{n_{y}}$, with known PDFs for the process noise, $p_{\mathbf{w}}(\mathbf{w})$, and measurement noise $p_{\mathbf{v}}(\mathbf{v})$. Let $\mathbb{Y}^{t}=\{\mathbf{y}(i)\}_{i=1}^{t}$ denote the set of all measurements up to time $t$.

The non-linear posterior prediction density $p(\mathbf{x}(t+$ 1) $\left.\mid \mathbb{Y}^{t}\right)$ and filtering density $p\left(\mathbf{x}(t) \mid \mathbb{Y}^{t}\right)$ for the Bayesian inference [15] are given by

$$
\begin{aligned}
p\left(\mathbf{x}(t+1) \mid \mathbb{Y}^{t}\right) & =\int_{\mathbb{R}^{n_{x}}} p(\mathbf{x}(t+1) \mid \mathbf{x}(t)) p\left(\mathbf{x}(t) \mid \mathbb{Y}^{t}\right) \mathrm{d} \mathbf{x}(t), \\
p\left(\mathbf{x}(t) \mid \mathbb{Y}^{t}\right) & =\frac{p(\mathbf{y}(t) \mid \mathbf{x}(t)) p\left(\mathbf{x}(t) \mid \mathbb{Y}^{t-1}\right)}{p\left(\mathbf{y}(t) \mid \mathbb{Y}^{t-1}\right)} .
\end{aligned}
$$


For the important special case of linear-Gaussian dynamics and linear-Gaussian observations, the Bayesian recursions in (12) have an analytical solution given by the KF, [17]. For non-linear and non-Gaussian systems, the PDF cannot, in general, be expressed with a finite number of parameters. Instead approximative methods are used.

For many non-linear problems, the noise assumptions and the system dynamics are such that a single Gaussian density approximates the underlying probability density sufficiently well. This is the idea behind the extended Kalman filter (EKF), [4,16], where the model is linearised around the previous estimate, and only the first and second order moments are estimated. The time update and measurement update for the EKF are given by

$$
\begin{aligned}
& \left\{\begin{array}{l}
\hat{\mathbf{x}}(t+1 \mid t)=f(\hat{\mathbf{x}}(t \mid t), \boldsymbol{\tau}(t), 0), \\
\mathbf{P}(t+1 \mid t)=\mathbf{F}(t) \mathbf{P}(t \mid t) \mathbf{F}(t)^{T}+\mathbf{G}(t) \mathbf{Q}(t) \mathbf{G}(t)^{T},
\end{array}\right. \\
& \left\{\begin{array}{l}
\hat{\mathbf{x}}(t \mid t)=\hat{\mathbf{x}}(t \mid t-1)+\mathbf{K}(t)(\mathbf{y}(t)-h(\hat{\mathbf{x}}(t \mid t-1))), \\
\mathbf{P}(t \mid t)=(\mathbf{I}-\mathbf{K}(t) \mathbf{H}(t)) \mathbf{P}(t \mid t-1), \\
\mathbf{K}(t)=\mathbf{P}(t \mid t-1) \mathbf{H}(t)^{T}\left(\mathbf{H}(t) \mathbf{P}(t \mid t-1) \mathbf{H}(t)^{T}+\mathbf{R}(t)\right)^{-1},
\end{array}\right.
\end{aligned}
$$

where the linearised matrices are given as

$$
\begin{aligned}
\mathbf{F}(t) & =\left.\nabla_{\mathbf{x}} f(\mathbf{x}(t), \boldsymbol{\tau}(t), 0)\right|_{\mathbf{x}(t)=\hat{\mathbf{x}}(t \mid t)} \\
\mathbf{G}(t) & =\left.\nabla_{\mathbf{w}} f(\mathbf{x}(t), \boldsymbol{\tau}(t), \mathbf{w}(t))\right|_{\mathbf{x}(t)=\hat{\mathbf{x}}(t \mid t)}, \\
\mathbf{H}(t) & =\left.\nabla_{\mathbf{x}} h(\mathbf{x}(t))\right|_{\mathbf{x}(t)=\hat{\mathbf{x}}(t \mid t-1)}
\end{aligned}
$$

The variables $\mathbf{P}(t+1 \mid t)$ and $\mathbf{P}(t \mid t)$ denote the covariance matrices for the estimation errors at time $t+1$ and $t$ given measurements up to time $t$, and the noise covariances are given as

$$
\mathbf{Q}(t)=\operatorname{Cov}(\mathbf{w}(t)), \mathbf{R}(t)=\operatorname{Cov}(\mathbf{v}(t)),
$$

where the process noise and measurement noise are assumed to be zero mean Gaussian distributions.

The KF is given by the same equations for the time and measurement update, where the system matrices $\mathbf{A}$, and $\mathbf{C}$ are obtained during the linearisation procedure with no need to use the previous estimate.

If a single Gaussian density does not approximate the posterior distribution sufficiently good, then simulation based methods such as the particle filter (PF) can be used to approximate the posterior PDF, see for instance [7]. In this paper only the EKF is used as an estimator, but Section 5.3 discusses briefly extensions to the control law based on the entire PDF, which could be implemented using a PF.

\section{Estimation-based ILC for Linear Systems}

The error $\mathbf{e}_{k}(t)$ used in the ILC algorithm is the difference between the reference $\mathbf{r}(t)$ and the controlled vari- able $\mathbf{z}_{k}(t)$ at iteration $k$. In general the controlled variable $\mathbf{z}_{k}(t)$ is not directly measurable, therefore the ILC algorithm has to rely on estimates based on measurements of related quantities. The situation is schematically described in Fig 1, where $\mathbf{r}(t)$ and $\mathbf{u}_{k}(t)$ denote the reference signal and the ILC input at iteration $k$, respectively. There are two types of output signals from the system, $\mathbf{z}_{k}(t)$ denotes the controlled variable and $\mathbf{y}_{k}(t)$ the measured variable at ILC iteration $k$. The system $\mathcal{S}$ can represent an open loop system as well as a closed loop system with internal feedback.

Let the error be given by

$$
\mathbf{e}_{k}(t)=\mathbf{r}(t)-\hat{\mathbf{z}}_{k}(t)
$$

where $\hat{\mathbf{z}}_{k}(t)$ is an estimate of the controlled variable. The estimate can be given by a single value, but often a Bayesian filter is used which gives the estimated quantity as a PDF $p(\mathbf{z})$ and the point estimate is given by $\hat{\mathbf{z}}_{k}(t)=$ $g(\mathbf{z}(t))$, where $\mathbf{z}(t) \sim p(\mathbf{z}(t))$ is a stochastic variable and $g(\cdot)$ is a function. Usually, the expected value $g(\cdot)=$ $\mathrm{E}[\cdot]$ is used, e.g. this is used in the EKF. If instead the $\mathrm{PF}$ is used, $\hat{\mathbf{z}}_{k}(t)$ can be chosen as e.g. the maximum a posteriori (MAP) estimate. Since the EKF provides both the mean estimate and the covariance matrix for the estimation error, and the PF provides an approximation of the posterior distribution $p\left(\mathbf{x}(t) \mid \mathbb{Y}^{t}\right)$ more information than only a point estimate such as the expected value can be used in the control law. In this section, only linear system will be treated and the KF is used for estimation. Section 5 extends the ideas to non-linear systems. It both cases it must be assumed that i) the system is observable, and ii) the filter, used for estimation, converges.

For evaluation of the performance of the ILC algorithm, the true error

$$
\varepsilon_{k}(t)=\mathbf{r}(t)-\mathbf{z}_{k}(t)
$$

is used.

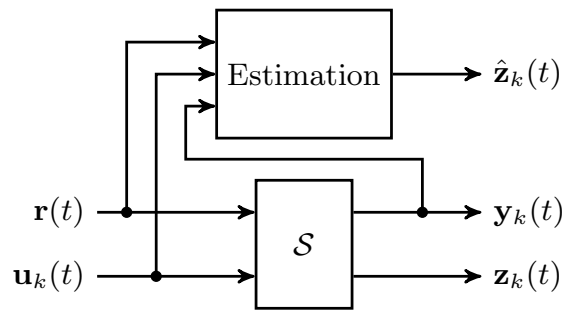

Fig. 1. System $\mathcal{S}$ with reference $\mathbf{r}(t)$, ILC input $\mathbf{u}_{k}(t)$, measured variable $\mathbf{y}_{k}(t)$ and controlled variable $\mathbf{z}_{k}(t)$ at ILC iteration $k$ and time $t$.

\subsection{Estimation-based Norm-optimal ILC}

The norm-optimal ILC from Section 2.1 can be extended to handle both the expected value and the covariance 
matrix of the estimated control error. Using a KF to estimate the state vector and calculating the control error according to $\mathbf{e}(t)=\mathbf{r}(t)-\hat{\mathbf{z}}(t)$ which gives that $\mathbf{e}(t)$ has a Gaussian distribution. Let the state vector of the system be estimated using a $\mathrm{KF}$, then it holds that

$$
\hat{\mathbf{x}}(t) \sim \mathcal{N}(\mathbf{x} ; \hat{\mathbf{x}}(t \mid t), \mathbf{P}(t \mid t))
$$

Moreover, let the estimated control variable be given by

$$
\hat{\mathbf{z}}(t)=\mathbf{C}_{z}(t) \hat{\mathbf{x}}(t)+\mathbf{D}_{z u}(t) \mathbf{u}(t)+\mathbf{D}_{z r}(t) \mathbf{r}(t) .
$$

Equation (17) is an affine transformation of a Gaussian distribution, hence

$$
\begin{aligned}
\hat{\mathbf{z}}(t) & \sim \mathcal{N}\left(\mathbf{z} ; \overline{\mathbf{z}}(t), \boldsymbol{\Sigma}_{\mathbf{z}}(t)\right) \\
\overline{\mathbf{z}}(t) & =\mathbf{C}_{z}(t) \hat{\mathbf{x}}(t \mid t)+\mathbf{D}_{z u}(t) \mathbf{u}(t)+\mathbf{D}_{z r}(t) \mathbf{r}(t) \\
\boldsymbol{\Sigma}_{\mathbf{z}}(t) & =\mathbf{C}_{\mathbf{z}}(t) \mathbf{P}(t \mid t) \mathbf{C}_{\mathbf{z}}(t)^{\top}
\end{aligned}
$$

Finally, the error $\mathbf{e}(t)=\mathbf{r}(t)-\hat{\mathbf{z}}(t)$ has the distribution

$$
\begin{aligned}
\mathbf{e}(t) & \sim p(\mathbf{e}(t))=\mathcal{N}\left(\mathbf{e} ; \overline{\mathbf{e}}(t), \boldsymbol{\Sigma}_{\mathbf{e}}(t)\right), \\
\overline{\mathbf{e}}(t) & =\mathbf{r}(t)-\overline{\mathbf{z}}(t), \\
\boldsymbol{\Sigma}_{\mathbf{e}}(t) & =\mathbf{C}_{\mathbf{z}}(t) \mathbf{P}(t \mid t) \mathbf{C}_{\mathbf{z}}(t)^{\top}
\end{aligned}
$$

\subsubsection{Choice of Objective Function}

A straightforward extension when the controlled signal $\mathbf{z}(t)$ is not measured but estimated, is to use the error $\mathbf{e}(t)=\mathbf{r}(t)-\mathrm{E}[\mathbf{z}(t)]$ in the norm-optimal ILC equations from Section 2.1 without considering the information provided in the covariance matrix. Another approach is to utilise the covariance matrix as follows: The objective of ILC is to achieve an error close to zero. Only considering the mean value is insufficient since a large variance means that there is a probability that the actual error is not close to zero. Hence, the mean of the distribution should be close to zero and at the same time the variance should be small. However, from the KF update equations it follows that the covariance matrix for the estimation error is independent of the observed data. It only depends on the model parameters and the initial value $\mathbf{P}_{0}$. Hence, it is not possible to affect the covariance of the error using $\mathbf{u}$. The covariance matrix is still a measure of how certain the estimated state is. A large covariance matrix indicates that the estimated state vector is uncertain. It is therefore natural to replace the term $\left\|\mathbf{e}_{k+1}(t)\right\|_{\mathbf{W}_{e}}^{2}$ with

$$
\left\|\overline{\mathbf{e}}_{k+1}(t)\right\|_{\boldsymbol{\Sigma}_{\mathbf{e}}^{-1}(t)}^{2}
$$

in (2). It means that if the estimated quantity is certain it will affect the objective function more than if it is less certain, i.e., an estimated error signal with large uncertainty has a low weight. The Lagrangian function becomes

$$
\begin{aligned}
\mathfrak{L}= & \frac{1}{2} \sum_{t=0}^{N-1}\left\|\overline{\mathbf{e}}_{k+1}(t)\right\|_{\boldsymbol{\Sigma}_{\mathbf{e}}^{-1}(t)}^{2} \\
& +\left\|\mathbf{u}_{k+1}(t)\right\|_{\mathbf{W}_{u}}^{2}+\lambda\left\|\mathbf{u}_{k+1}(t)-\mathbf{u}_{k}(t)\right\|^{2}
\end{aligned}
$$

\subsubsection{Solution of the Optimisation Problem}

The Lagrangian function (21) in matrix form is given by

$$
\begin{array}{r}
\mathfrak{L}=\frac{1}{2}\left[\overline{\mathbf{e}}_{k+1}^{\top} \mathcal{W}_{\mathbf{e}} \overline{\mathbf{e}}_{k+1}+\mathbf{u}_{k+1}^{\top} \mathcal{W}_{\mathbf{u}} \mathbf{u}_{k+1}\right. \\
\left.+\lambda\left(\mathbf{u}_{k+1}-\mathbf{u}_{k}\right)^{\top}\left(\mathbf{u}_{k+1}-\mathbf{u}_{k}\right)\right]
\end{array}
$$

where

$$
\begin{aligned}
\overline{\mathbf{e}}_{k} & =\left(\overline{\mathbf{e}}_{k}(0)^{\top} \ldots \overline{\mathbf{e}}_{k}(N-1)^{\top}\right)^{\top} \in \mathbb{R}^{N n_{z}} \\
\mathcal{W}_{\mathbf{e}} & =\operatorname{diag}\left(\boldsymbol{\Sigma}_{\mathbf{e}}^{-1}(0), \ldots, \boldsymbol{\Sigma}_{\mathbf{e}}^{-1}(N-1)\right) \in \mathbb{S}_{++}^{N n_{z}}
\end{aligned}
$$

Let the state space model be given by

$$
\begin{aligned}
\mathbf{x}_{k}(t+1) & =\mathbf{A}(t) \mathbf{x}_{k}(t)+\mathbf{B}_{u} \mathbf{u}_{k}(t)+\mathbf{B}_{r} \mathbf{r}(t) \\
\mathbf{y}_{k}(t) & =\mathbf{C}_{\mathbf{y}}(t) \mathbf{x}_{k}(t)+\mathbf{D}_{\mathbf{y u}}(t) \mathbf{u}_{k}(t)+\mathbf{D}_{\mathbf{y r}}(t) \mathbf{r}(t) \\
\mathbf{z}_{k}(t) & =\mathbf{C}_{\mathbf{z}}(t) \mathbf{x}_{k}(t)+\mathbf{D}_{\mathbf{z u}}(t) \mathbf{u}_{k}(t)+\mathbf{D}_{\mathbf{z r}}(t) \mathbf{r}(t)
\end{aligned}
$$

then the output $\mathbf{y}_{k}$ and the estimate $\hat{\mathbf{z}}_{k}$ in batch form becomes

$$
\begin{aligned}
& \mathbf{y}_{k}=\mathcal{C}_{\mathbf{y}} \boldsymbol{\Phi} \mathbf{x}_{0}+\mathbf{T}_{\mathbf{y u}} \mathbf{u}_{k}+\mathbf{T}_{\mathbf{y} \mathbf{r}} \mathbf{r} \\
& \hat{\mathbf{z}}_{k}=\mathcal{C}_{\mathbf{z}} \widetilde{\boldsymbol{\Phi}} \hat{\mathbf{x}}_{0}+\mathbf{T}_{\hat{\mathbf{z}} \mathbf{u}} \mathbf{u}_{k}+\mathbf{T}_{\hat{\mathbf{z}} \mathbf{r}} \mathbf{r}+\mathbf{T}_{\hat{\mathbf{z}} \mathbf{y}} \mathbf{y}_{k}
\end{aligned}
$$

where the KF recursion in batch form has been applied. See Appendix A for details. The Lagrangian function (22) using $\overline{\mathbf{e}}_{k+1}=\mathbf{r}-\hat{\mathbf{z}}_{k+1}$ becomes

$$
\begin{aligned}
\mathfrak{L}= & \frac{1}{2} \mathbf{u}_{k+1}^{\top}\left(\mathbf{T}_{\mathbf{u}}^{\top} \mathcal{W}_{\mathbf{e}} \mathbf{T}_{\mathbf{u}}+\mathcal{W}_{\mathbf{u}}+\lambda \mathbf{I}\right) \mathbf{u}_{k+1} \\
+ & {\left[\left(\left(\mathbf{I}-\mathbf{T}_{\mathbf{r}}\right) \mathbf{r}-\mathcal{C}_{\mathbf{z}} \widetilde{\mathbf{\Phi}} \hat{\mathbf{x}}_{0}-\mathbf{T}_{\hat{\mathbf{z}} \mathbf{y}} \mathcal{\mathcal { C } _ { \mathbf { y } }} \mathbf{\Phi} x_{0}\right)^{\top} \mathcal{W}_{\mathbf{e}} \mathbf{T}_{\mathbf{u}}\right.} \\
& \left.+\lambda \mathbf{u}_{k}^{\top}\right] \mathbf{u}_{k+1}
\end{aligned}
$$

where $\mathbf{T}_{\mathbf{u}}=\mathbf{T}_{\hat{\mathbf{z}} \mathbf{u}}+\mathbf{T}_{\hat{\mathbf{z} y} \mathbf{y}} \mathbf{T}_{\mathbf{y u}}, \mathbf{T}_{\mathbf{r}}=\mathbf{T}_{\hat{\mathbf{z}} \mathbf{r}}+\mathbf{T}_{\hat{\mathbf{z}} \mathbf{y}} \mathbf{T}_{\mathbf{y r} \mathbf{r}}$, and all the terms independent of $\mathbf{u}_{k+1}$ are omitted.

The same arguments as in Section 2.1 result in a positive definite Hessian matrix of $\mathfrak{L}$ in (25), hence taking the derivative of $\mathfrak{L}$ with respect to $\mathbf{u}_{k+1}$ equal to zero gives 
the optimal ILC control signal

$$
\begin{aligned}
\mathbf{u}_{k+1}= & \mathcal{Q} \cdot\left(\mathbf{u}_{k}+\mathcal{L} \cdot \overline{\mathbf{e}}_{k}\right) \\
\mathcal{Q}= & \left(\mathbf{T}_{\mathbf{u}}^{\top} \mathcal{W}_{\mathbf{e}} \mathbf{T}_{\mathbf{u}}+\mathcal{W}_{\mathbf{u}}+\lambda \mathbf{I}\right)^{-1} \\
& \times\left(\lambda \mathbf{I}+\mathbf{T}_{\mathbf{u}}^{\top} \mathcal{W}_{\mathbf{e}} \mathbf{T}_{\mathbf{u}}\right) \\
\mathcal{L}= & \left(\lambda \mathbf{I}+\mathbf{T}_{\mathbf{u}}^{\top} \mathcal{W}_{\mathbf{e}} \mathbf{T}_{\mathbf{u}}\right)^{-1} \mathbf{T}_{\mathbf{u}}^{\top} \mathcal{W}_{\mathbf{e}}
\end{aligned}
$$

where $\overline{\mathbf{e}}_{k}=\mathbf{r}-\hat{\mathbf{z}}_{k}$, (23) and (24) have been used to eliminate the terms involving $\mathbf{r}, \mathbf{x}_{0}, \hat{\mathbf{x}}_{0}$, and $\mathbf{y}_{k}$.

The expressions for $\mathcal{Q}$ and $\mathcal{L}$ in (26) are similar to (8). The only difference is that $\mathbf{T}_{\mathbf{u}}$ is used instead of $\mathbf{T}_{\mathbf{z u}}$. For the special case of LTI-systems using the stationary $\mathrm{KF}^{3}$ it can be shown that the KF does not affect the solution. This is presented in Theorem 2

Theorem 2 (Separation lemma for estimationbased $\mathbf{I L C}$ ): Given an LTI-system and the stationary $\mathrm{KF}$ with gain matrix $\mathbf{K}$, then the matrices $\mathbf{T}_{\mathbf{u}}$ and $\mathbf{T}_{\mathbf{z u}}$ are equal, hence the ILC update law (8) can be derived without considering the $\mathrm{KF}$ estimator.

PROOF. It follows from the expressions of $\mathbf{T}_{\mathbf{z u}}$ and $\mathbf{T}_{\mathbf{u}}$ using the constant state space matrices and a constant Kalman gain.

The result from Theorem 2 makes it more computational efficient to compute the matrices $\mathcal{Q}$ and $\mathcal{L}$, since the matrix $\mathbf{T}_{\mathbf{z u}}$ requires fewer calculations to obtain, compared to the matrix $\mathbf{T}_{\mathbf{u}}$. Even if the iterative KF update is used, the Kalman gain $\mathbf{K}$ converges eventually to the stationary value for LTI systems. If this is done reasonably fast, the approximation $\mathbf{T}_{\mathbf{u}} \approx \mathbf{T}_{\mathbf{z u}}$ can be good enough to use.

\subsubsection{Stability Analysis}

Assuming that the KF is initialised with the same covariance matrix $\mathbf{P}_{0}$ at each iteration, makes the sequence of Kalman gains $\mathbf{K}(t), t=0, \ldots, N-1$ the same for different ILC iterations since $\mathbf{P}$ and $\mathbf{K}$ are independent of the control signal $\mathbf{u}$. The system matrices are therefore constant over the ILC iterations, hence the same analysis for stability and monotone convergence as in Section 2.2 can be applied. The iterative system for $\mathbf{u}_{k}$ is given by

$$
\begin{aligned}
\mathbf{u}_{k+1}= & \mathcal{Q} \cdot\left(\mathbf{I}-\mathcal{L} \cdot \mathbf{T}_{\mathbf{u}}\right) \mathbf{u}_{k}+\mathcal{Q} \cdot \mathcal{L} \cdot\left(\mathbf{I}-\mathbf{T}_{\mathbf{r}}\right) \mathbf{r} \\
& -\mathcal{Q} \cdot \mathcal{L} \cdot\left(\mathcal{C}_{\mathbf{z}} \widetilde{\mathbf{\Phi}}_{\mathbf{x}_{0}}+\mathbf{T}_{\hat{\mathbf{z}} \mathbf{y}} \mathcal{C}_{\mathbf{y}} \mathbf{\Phi} \mathbf{x}_{0}\right)
\end{aligned}
$$

\footnotetext{
3 The stationary Kalman filter uses a constant gain $\mathbf{K}$ which is the solution to an algebraic Riccati equation, see [4].
}

where (26a), (24), (23), and $\overline{\mathbf{e}}_{k}=\mathbf{r}-\hat{\mathbf{z}}_{k}$ have been used. The results for stability and monotone convergence for the ILC update law (26) is given in Theorem 3.

Theorem 3 (Stability and monotonic convergence): The iterative system (27) is stable and monotonically convergent for all system descriptions in (24), and (23) using $\mathcal{Q}$ and $\mathcal{L}$ from (26).

PROOF. The proof is the same as for Theorem 1 with the variable change $\mathbf{T}_{\mathbf{z u}} \rightarrow \mathbf{T}_{\mathbf{u}}$.

The asymptotic control signal $\mathbf{u}_{\infty}$ and asymptotic error $\mathbf{e}_{\infty}$ can be obtained similar to what is discussed in Section 2.2 .

\subsection{Estimation using Internal Signals}

In the derivation of the estimation-based norm-optimal ILC update law in Section 4.1 it is assumed that the KF takes the signals $\mathbf{r}(t)$ and $\mathbf{u}_{k}(t)$ as inputs. In general the estimation problem does not work with $\mathbf{r}(t)$ and $\mathbf{u}_{k}(t)$. As an example, a system with a feedback loop uses the input to the controlled system for estimation, not the input to the controller. More general, the estimation problem only uses a part of the full system $\mathcal{S}$ for estimation, whereas the other part is completely known or not interesting to use for estimation. Nevertheless, the structure in Fig 1 is a valid description of the estimation-based ILC.

Let $\boldsymbol{\tau}_{k}(t)$ be the known signal used for estimation, hence the estimated variable can be written as

$$
\hat{\mathbf{z}}_{k}(t)=F_{\hat{\mathbf{z}} \boldsymbol{\tau}}(q) \boldsymbol{\tau}_{k}(t)+F_{\hat{\mathbf{z}} \mathbf{y}}(q) \mathbf{y}_{k}(t) .
$$

However, the signal $\boldsymbol{\tau}_{k}(t)$ can be seen as an output from a system with $\mathbf{r}(t), \mathbf{u}_{k}(t)$, and $\mathbf{y}_{k}(t)$ as inputs, hence

$$
\boldsymbol{\tau}_{k}(t)=F_{\boldsymbol{\tau} \mathbf{r}}(q) \mathbf{r}(t)+F_{\boldsymbol{\tau} \mathbf{u}}(q) \mathbf{u}_{k}(t)+F_{\boldsymbol{\tau} \mathbf{y}}(q) \mathbf{y}_{k}(t) .
$$

Combining (28) and (29) gives

$$
\hat{\mathbf{z}}_{k}(t)=T_{\hat{\mathbf{z}} \mathbf{r}}(q) \mathbf{r}(t)+T_{\hat{\mathbf{z}} \mathbf{u}}(q) \mathbf{u}_{k}(t)+T_{\hat{\mathbf{z}} \mathbf{y}}(q) \mathbf{y}_{k}(t),
$$

where

$$
\begin{aligned}
& T_{\hat{\mathbf{z}} \mathbf{r}}(q)=F_{\hat{\mathbf{z}} \boldsymbol{\tau}}(q) F_{\boldsymbol{\tau} \mathbf{r}}(q), T_{\hat{\mathbf{z}} \mathbf{u}}(q)=F_{\hat{\mathbf{z}} \boldsymbol{\tau}}(q) F_{\boldsymbol{\tau} \mathbf{u}}(q) \\
& T_{\hat{\mathbf{z}} \mathbf{y}}(q)=F_{\hat{\mathbf{z}} \boldsymbol{\tau}}(q) F_{\boldsymbol{\tau} \mathbf{y}}(q)+F_{\hat{\mathbf{z}} \mathbf{y}}(q)
\end{aligned}
$$

which is in the form described in Fig 1. It is straightforward to take this into consideration when deriving the ILC update control law and it changes only the definition of $\mathbf{T}_{\hat{\mathbf{z}} \mathbf{u}}, \mathbf{T}_{\hat{\mathbf{z}} \mathbf{r}}$, and $\mathbf{T}_{\hat{\mathbf{z}} \mathbf{y}}$. Note that the dimension of the 
state vector describing (23) and (24) can differ since (24) is constructed using only a part of the complete system $\mathcal{S}$.

\section{Estimation-based ILC for Non-linear Systems}

The estimation-based norm-optimal ILC in Section 4.1 can be extended to non-linear systems. The minimisation problem is the same as in (2) but the dynamics and measurements are given by non-linear functions, hence the minimisation problem has to be solved using a numerical solver. However, an estimate of the complete state trajectory is given from the estimation step at the previous ILC iteration which can be used for linearisation of the non-linear state space model. The only necessary assumption is to have $\mathbf{u}_{k+1}$ close to $\mathbf{u}_{k}$ which is possible to achieve by assigning $\lambda$ a large enough value. The drawback is that the convergence rate can become slow.

Sections 5.1 and 5.2 discuss the solution and stability analysis for the linearised model, and Section 5.3 discusses briefly possible extensions without performing any linearisation.

\subsection{Solution using Linearised Model}

The non-linear model

$$
\begin{aligned}
& \mathbf{x}_{k}(t+1)=f\left(\mathbf{x}_{k}(t), \mathbf{u}_{k}(t), \mathbf{r}(t)\right) \\
& \mathbf{y}_{k}(t+1)=h_{\mathbf{y}}\left(\mathbf{x}_{k}(t), \mathbf{u}_{k}(t), \mathbf{r}(t)\right) \\
& \mathbf{z}_{k}(t+1)=h_{\mathbf{z}}\left(\mathbf{x}_{k}(t), \mathbf{u}_{k}(t), \mathbf{r}(t)\right)
\end{aligned}
$$

is linearised around $\hat{\mathbf{x}}_{k-1}(t \mid t), \mathbf{u}_{k-1}(t)$, and $\mathbf{r}(t)$ giving the linear model

$$
\begin{aligned}
\mathbf{x}_{k}(t+1) & =\mathbf{A}_{k-1}(t) \mathbf{x}_{k}(t)+\mathbf{B}_{k-1}(t) \mathbf{u}_{k}(t)+\mathbf{s}_{\mathbf{x}, k-1}(t) \\
\mathbf{y}_{k}(t) & =\mathbf{C}_{\mathbf{y}, k-1} \mathbf{x}_{k}(t)+\mathbf{D}_{\mathbf{y}, k-1}(t) \mathbf{u}_{k}(t)+\mathbf{s}_{\mathbf{y}, k-1}(t) \\
\mathbf{z}_{k}(t) & =\mathbf{C}_{\mathbf{z}, k-1} \mathbf{x}_{k}(t)+\mathbf{D}_{\mathbf{z}, k-1}(t) \mathbf{u}_{k}(t)+\mathbf{s}_{\mathbf{z}, k-1}(t)
\end{aligned}
$$

where

$$
\begin{aligned}
& \mathbf{A}_{k-1}(t)=\left.\frac{\partial f}{\partial \mathbf{x}}\right|_{\substack{\mathbf{x}=\hat{\mathbf{x}}_{k-1}(t \mid t) \\
\mathbf{u}=\mathbf{u}_{k-1}(t) \\
\mathbf{r}=\mathbf{r}(t)}}, \quad \mathbf{B}_{k-1}(t)=\left.\frac{\partial f}{\partial \mathbf{u}}\right|_{\substack{\mathbf{x}=\hat{\mathbf{x}}_{k-1}(t \mid t) \\
\mathbf{u}=\mathbf{u}_{k-1}(t) \\
\mathbf{r}=\mathbf{r}(t)}} \\
& \mathbf{C}_{\mathbf{y}, k-1}(t)=\left.\frac{\partial h_{\mathbf{y}}}{\partial \mathbf{x}}\right|_{\substack{\mathbf{r}=\hat{\mathbf{x}}_{k-1}(t \mid t) \\
\mathbf{u}=\mathbf{u}_{k-1}(t) \\
\mathbf{r}=\mathbf{r}(t)}} ^{\mathbf{r}=\mathbf{r}(t)}, \mathbf{C}_{\mathbf{z}, k-1}(t)=\left.\frac{\partial h_{\mathbf{z}}}{\partial \mathbf{x}}\right|_{\substack{\mathbf{x}=\hat{\mathbf{x}}_{k-1}(t \mid t) \\
\mathbf{u}=\mathbf{u}_{k-1}(t) \\
\mathbf{r}=\mathbf{r}(t)}} ^{\mathbf{r}=\mathbf{r}(t)} \\
& \mathbf{D}_{\mathbf{y}, k-1}(t)=\left.\frac{\partial h_{\mathbf{y}}}{\partial \mathbf{u}}\right|_{\substack{\mathbf{x}=\hat{\mathbf{x}}_{k-1}(t \mid t) \\
\mathbf{u}=\mathbf{u}_{k-1}(t) \\
\mathbf{r}=\mathbf{r}(t)}} ^{\mathbf{r}=\mathbf{r}(t)}, \mathbf{D}_{\mathbf{z}, k-1}(t)=\left.\frac{\partial h_{\mathbf{z}}}{\partial \mathbf{u}}\right|_{\substack{\mathbf{x}=\hat{\mathbf{x}}_{k-1}(t \mid t) \\
\mathbf{u}=\mathbf{u}_{k-1}(t) \\
\mathbf{r}=\mathbf{r}(t)}} ^{\mathbf{r}=\mathbf{r}(t)}
\end{aligned}
$$

$$
\begin{aligned}
\mathbf{s}_{\mathbf{x}, k-1}(t)= & f\left(\hat{\mathbf{x}}_{k-1}(t \mid t), \mathbf{u}_{k-1}(t), \mathbf{r}(t)\right) \\
& -\mathbf{A}_{k-1}(t) \hat{\mathbf{x}}_{k-1}(t \mid t)-\mathbf{B}_{k-1}(t) \mathbf{u}_{k-1}(t) \\
\mathbf{s}_{\mathbf{y}, k-1}(t)= & h_{y}\left(\hat{\mathbf{x}}_{k-1}(t \mid t), \mathbf{u}_{k-1}(t), \mathbf{r}(t)\right) \\
& -\mathbf{C}_{\mathbf{y}, k-1}(t) \hat{\mathbf{x}}_{k-1}(t \mid t)-\mathbf{D}_{\mathbf{y}, k-1}(t) \mathbf{u}_{k-1}(t) \\
\mathbf{s}_{\mathbf{z}, k-1}(t)= & h_{z}\left(\hat{\mathbf{x}}_{k-1}(t \mid t), \mathbf{u}_{k-1}(t), \mathbf{r}(t)\right) \\
& -\mathbf{C}_{\mathbf{z}, k-1}(t) \hat{\mathbf{x}}_{k-1}(t \mid t)-\mathbf{D}_{\mathbf{z}, k-1}(t) \mathbf{u}_{k-1}(t)
\end{aligned}
$$

Here, the matrices and s-variables only depend on the previous ILC iteration, hence they are known at the current iteration. Using the linearised state space model gives, as before, the estimate $\hat{\mathbf{z}}_{k}(t)$ and the output $\mathbf{y}_{k}(t)$ in batch form

$$
\begin{aligned}
\mathbf{y}_{k}= & \mathcal{C}_{\mathbf{y}, k-1} \boldsymbol{\Phi}_{k-1} \mathbf{x}_{0}+\mathbf{T}_{\mathbf{y u}, k-1} \mathbf{u}_{k}+\mathbf{T}_{\mathbf{y} \mathbf{s}_{\mathbf{x}}, k-1} \mathbf{s}_{\mathbf{x}, k-1} \\
& +\mathbf{s}_{\mathbf{y}, k-1} \\
\hat{\mathbf{z}}_{k}= & \mathcal{C}_{\mathbf{z}, k-1} \widetilde{\mathbf{\Phi}}_{k-1} \hat{\mathbf{x}}_{0}+\mathbf{T}_{\hat{\mathbf{z}} \mathbf{u}, k-1} \mathbf{u}_{k}+\mathbf{T}_{\hat{\mathbf{z}} \mathbf{y}, k-1} \mathbf{y}_{k} \\
& +\mathbf{T}_{\hat{\mathbf{z}} \mathbf{s}_{\mathbf{x}}, k-1} \mathbf{s}_{\mathbf{x}, k-1}+\mathbf{T}_{\hat{\mathbf{z}} \mathbf{S}_{\mathbf{y}}, k-1} \mathbf{s}_{\mathbf{y}, k-1}+\mathbf{s}_{\mathbf{z}, k-1}
\end{aligned}
$$

where all the matrices in the right hand side are dependent of $k$, and the vectors $\mathbf{s}_{\mathbf{x}, k}, \mathbf{s}_{\mathbf{y}, k}$, and $\mathbf{s}_{\mathbf{z}, k}$ are stacked versions of $\mathbf{s}_{\mathbf{x}, k}(t), \mathbf{s}_{\mathbf{y}, k}(t)$, and $\mathbf{s}_{\mathbf{z}, k}(t)$.

The norm-optimal ILC problem can be formulated and solved in the same way as described in Section 4.1.2. Unfortunately, since the batch form matrices are dependent of $k$, the last step of the solution where the terms including $\mathbf{r}, \hat{\mathbf{x}}_{0}$ and $\mathbf{x}_{0}$ are removed, cannot be performed. Here, the terms including $\mathbf{s}_{\mathbf{x}, k}, \mathbf{s}_{\mathbf{y}, k}$, and $\mathbf{s}_{\mathbf{z}, k}$ will also remain. Note that the weight matrix for the error is also dependent of $k$, since it consists of the covariance matrices for the control error. The solution is given by

$$
\begin{aligned}
\mathbf{u}_{k+1}= & \left(\mathbf{T}_{\mathbf{u}, k}^{\top} \mathcal{W}_{\mathbf{e}, k} \mathbf{T}_{\mathbf{u}, k}+\mathcal{W}_{\mathbf{u}}+\lambda \mathbf{I}\right)^{-1}\left[\lambda \mathbf{u}_{k}\right. \\
& +\mathbf{T}_{\mathbf{u}, k}^{\top} \mathcal{W}_{\mathbf{e}, k}\left(\mathbf{r}-\mathcal{C}_{\mathbf{z}, k} \widetilde{\boldsymbol{\Phi}}_{k} \hat{\mathbf{x}}_{0}-\mathbf{T}_{\hat{\mathbf{z}} \mathbf{s}_{\mathbf{x}}, k} \mathbf{s}_{\mathbf{x}, k}\right. \\
& -\mathbf{T}_{\hat{\mathbf{z}} \mathbf{s}_{\mathbf{y}}, k} \mathbf{s}_{\mathbf{y}, k}-\mathbf{s}_{\mathbf{z}, k}-\mathbf{T}_{\hat{\mathbf{z}} \mathbf{y}, k}\left(\mathcal{C}_{\mathbf{y}, k} \mathbf{\Phi}_{k} \mathbf{x}_{0}\right. \\
& \left.\left.\left.+\mathbf{T}_{\mathbf{y} \mathbf{s}_{\mathbf{x}}, k} \mathbf{s}_{\mathbf{x}, k}+\mathbf{s}_{\mathbf{y}, k}\right)\right)\right]
\end{aligned}
$$

where $\mathbf{T}_{\mathbf{u}, k}=\mathbf{T}_{\hat{\mathbf{z}} \mathbf{u}, k}+\mathbf{T}_{\hat{\mathbf{z} y}, k} \mathbf{T}_{\mathbf{y u}, k}$. The initial condition $\mathbf{x}_{0}$ is of course not known, hence $\hat{\mathbf{x}}_{0}$ must be used instead.

If the change in $\mathbf{u}$ is sufficiently small, i.e., $\left\|\mathbf{u}_{k+1}-\mathbf{u}_{k}\right\|$ is small enough, then the approximation $\mathbf{T}_{\mathbf{y u}, k-1} \approx$ $\mathbf{T}_{\mathbf{y u}, k}$ and similar for the rest of the matrices and the $\mathbf{s}$-variables can be appropriate. Note that the change in $\mathbf{u}$ depends strongly on the choice of $\lambda$. If that is the case then the ILC update law (32) is simplified to

$$
\begin{aligned}
\mathbf{u}_{k+1}= & \mathcal{Q}_{k} \cdot\left(\mathbf{u}_{k}+\mathcal{L}_{k} \cdot \overline{\mathbf{e}}_{k}\right) \\
\mathcal{Q}_{k}= & \left(\mathbf{T}_{\mathbf{u}, k}^{\boldsymbol{T}} \mathcal{W}_{\mathbf{e}, k} \mathbf{T}_{\mathbf{u}, k}+\mathcal{W}_{\mathbf{u}}+\lambda \mathbf{I}\right)^{-1} \\
& \times\left(\lambda \mathbf{I}+\mathbf{T}_{\mathbf{u}, k}^{\boldsymbol{T}} \mathcal{W}_{\mathbf{e}, k} \mathbf{T}_{\mathbf{u}, k}\right) \\
\mathcal{L}_{k}= & \left(\lambda \mathbf{I}+\mathbf{T}_{\mathbf{u}, k}^{\boldsymbol{\top}} \mathcal{W}_{\mathbf{e}, k} \mathbf{T}_{\mathbf{u}, k}\right)^{-1} \mathbf{T}_{\mathbf{u}, k}^{\boldsymbol{T}} \mathcal{W}_{\mathbf{e}, k}
\end{aligned}
$$




\subsection{Stability Analysis for the Linearised Solution}

For non-linear system, utilising the above described linearisation technique, gives ILC iteration-variant system descriptions. Hence, stability cannot be ensured as for the linear system previously analysed. Instead the convergence results from [23], which are based on theory for discrete time-variant systems, has to be used. The stability properties for the iteration-variant ILC system (32) is presented in Theorem 4.

Theorem 4 (Iteration-variant stability): The ILC system (32) is stable for all linearised systems.

PROOF. From [23, Corollary 3] it follows that the ILC system (32) is stable iff

$$
\bar{\sigma}\left(\left(\mathbf{T}_{\mathbf{u}, k}^{\top} \mathcal{W}_{\mathbf{e}, k} \mathbf{T}_{\mathbf{u}, k}+\mathcal{W}_{\mathbf{u}}+\lambda \mathbf{I}\right)^{-1} \lambda\right)<1
$$

for all $k$. Moreover, it holds that the weight matrix $\mathcal{W}_{\mathbf{e}, k} \in \mathbb{S}_{++}$for all $k$, due to the construction from the covariance matrices. This fact, together with the fact that $\mathcal{W}_{\mathbf{u}} \in \mathbb{S}_{++}$, and $\lambda \in \mathbb{R}_{+}$guarantee that (34) is fulfilled for all $k$, hence the ILC system is stable.

\subsection{Extension without Linearisation}

If no linearisation can be used, or if the estimate is given by a PF, then the objective function can be modified using e.g. the Kullback-Leibler (KL) divergence [18]. As discussed in Section 4.1.1 it is desirable to have the mean of the distribution of the control error close to zero and at the same time having a small variance. One way to measure this is to compare the PDF of the error with a desired PDF with zero mean and small variance. One such measure is the KL-divergence, which for the two PDFs $p(\mathbf{x})$ and $q(\mathbf{x})$ is defined as

$$
D_{\mathrm{KL}}(p(\mathbf{x}) \| q(\mathbf{x})) \triangleq \int_{\mathbb{R}^{n_{x}}} p(\mathbf{x}) \log \frac{p(\mathbf{x})}{q(\mathbf{x})} \mathrm{d} \mathbf{x} \geq 0
$$

with equality if and only if $p(\mathbf{x})=q(\mathbf{x})$. Moreover, the KL-divergence is not symmetric, i.e., $D_{\mathrm{KL}}(p(\mathbf{x}) \| q(\mathbf{x})) \neq$ $D_{\mathrm{KL}}(q(\mathbf{x}) \| p(\mathbf{x}))$. The KL-divergence is closely related to other statistical measures, e.g., Shannon's information and Akaike's information criterion [6]. A connection to Fisher information can also be found [18]. The objective function (2) is modified by replacing the term $\left\|\mathbf{e}_{k+1}(t)\right\|_{\mathbf{W}_{\mathbf{e}}}^{2}$ with the KL-divergence $D_{\mathrm{KL}}\left(q\left(\mathbf{e}_{k+1}(t)\right) \| p\left(\mathbf{e}_{k+1}(t)\right)\right)$, where $p\left(\mathbf{e}_{k+1}(t)\right)$ is the actual distribution of the error given by the estimator, and $q\left(\mathbf{e}_{k+1}(t)\right)$ is the desired distribution for the error, which is to be chosen.
Although, using this idea is outside the scope of this work. It can be noted that, using the KL-divergence for the linear case where $\mathbf{e}_{k}(t)$ is Gaussian distributed, see Section 4.1, gives exactly the same Lagrangian function as described in Section 4.1.1. It follows from the fact that if $p(\mathbf{e}(t))$ is Gaussian then it is reasonable to assume that it should resemble $q(\mathbf{e}(t))=\mathcal{N}(\mathbf{e} ; \mathbf{0}, \boldsymbol{\Sigma})$, where $\boldsymbol{\Sigma}$ is a, not too large, constant to choose. Straightforward calculations utilizing two Gaussian distributions, see [6] for details, give the following KL-divergence

$$
\begin{aligned}
& D_{\mathrm{KL}}\left(\mathcal{N}\left(\mathbf{x} ; \boldsymbol{\mu}_{0}, \boldsymbol{\Sigma}_{0}\right) \| \mathcal{N}\left(\mathbf{x} ; \boldsymbol{\mu}_{1}, \boldsymbol{\Sigma}_{1}\right)\right)=\frac{1}{2}\left(\operatorname{tr} \boldsymbol{\Sigma}_{2}^{-1} \boldsymbol{\Sigma}_{1}\right. \\
& \left.+\left(\boldsymbol{\mu}_{1}-\boldsymbol{\mu}_{2}\right)^{\top} \boldsymbol{\Sigma}_{2}^{-1}\left(\boldsymbol{\mu}_{1}-\boldsymbol{\mu}_{2}\right)+\log \frac{\left|\boldsymbol{\Sigma}_{2}\right|}{\left|\boldsymbol{\Sigma}_{1}\right|}-n_{x}\right)
\end{aligned}
$$

where $\operatorname{tr}$ is the trace operator and $|\cdot|$ the determinant of a matrix. With $p(\mathbf{e}(t))$ from $(19)$ and $q(\mathbf{e}(t))=\mathcal{N}(\mathbf{e} ; \mathbf{0}, \boldsymbol{\Sigma})$ give

$$
D_{\mathrm{KL}}(q(\mathbf{e}(t)) \| p(\mathbf{e}(t)))=\frac{1}{2} \overline{\mathbf{e}}(t)^{\top} \boldsymbol{\Sigma}_{\mathbf{e}}^{-1}(t) \overline{\mathbf{e}}(t)+\xi,
$$

where $\xi$ is constant with respect to $\mathbf{x}, \mathbf{u}$, and $\mathbf{y}$. The expression in (37) is the same as the one in (20). By interchanging the distributions $p$ and $q$ the result will be the norm of the mean error but now weighted with $\boldsymbol{\Sigma}^{-1}$, which is a tuning parameter, hence no information of the covariance matrix of the control error is used.

\section{Simulation Results}

The norm-optimal ILC control law for non-linear systems, described in Section 5.1 is evaluated on a flexible single joint model, see Fig 2, where the estimation is performed using an EKF. The single joint model is based on the two-axes model in [20], where the model structure and the parameters are presented. To get a single joint model, the second joint for the two-axes model has been positioned in $-\pi / 2$ and it is assumed rigidly attached to the first arm. The link is modelled as a rigid-body and the joint flexibility is modelled as a spring damping pair with non-linear spring torque and linear damping. Finally, the motor characteristics are given by a non-linear friction torque.

Using the state vector $\mathbf{x}=\left(\begin{array}{llll}q_{a} & q_{m} & \dot{q}_{a} & \dot{q}_{m}\end{array}\right)^{\top}$, where $q_{a}$ is the arm angle and $q_{m}$ the motor angle, and the dynamical model gives a non-linear state space model according to $\dot{\mathbf{x}}=\tilde{f}(\mathbf{x}, \tau)$. The continuous-time model is discretised using Euler sampling giving the discrete-time model $\mathbf{x}(t+1)=f(\mathbf{x}(t), \tau(t))$ which is used in the EKF. The model also includes zero mean Gaussian distributed process noise with the variance $Q=10^{6}$. The process noise enters the model in the same way the motor torque $\tau(t)$ does. The sample time is $T_{s}=1 \mathrm{~ms}$. 


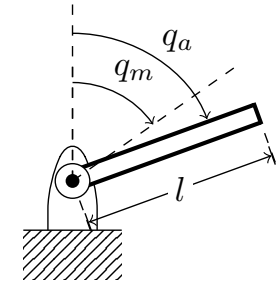

Fig. 2. A single flexible joint corresponding to joint two of a six-axes industrial manipulator. The deflection is described by the difference of the arm angle $q_{a}$ and the motor angle $q_{m}$.

The measurements used in the EKF are the motor angle $q_{m}$ and the acceleration of the tool position $l \ddot{q}_{a}$, where $l$ is the length of the arm and $\ddot{q}_{a}$ is the arm angular acceleration. Additive zero mean Gaussian measurement noise with covariance matrix $\mathbf{R}=10^{-4} \mathbf{I}$ is also included.

The joint model is stabilised using a P-controller with gain 1, hence the ILC control law from Section 5.1 has to be modified according to Section 4.2 . Note that the $\mathrm{P}$-controller is only included to stabilise the system, not give a good closed loop performance.

The reference signal $r(t)$ for the arm angle is chosen as a step filtered through a FIR filter of length $n=100$ with all coefficients equal to $1 / n$. The filtering is performed in four consecutive steps to get a twice differentiable smooth reference signal. The reference signal for the motor angle, used for the feedback loop, is, for simplicity, taken to be the same as the arm angle reference $r(t)$.

The performance of the ILC algorithm is evaluated using the relative reduction of the RMSE in percentage with respect to the error when no ILC signal is applied

$$
\rho_{k}=100 \sqrt{\frac{1}{N} \sum_{t=1}^{N-1} \varepsilon_{k}(t)^{2}} / \sqrt{\frac{1}{N} \sum_{t=1}^{N-1} \varepsilon_{0}(t)^{2}},
$$

where $\varepsilon_{k}(t)$ is given by $(15)$.

The estimation-based norm-optimal ILC control law in (33) is used with the five different settings of $\mathbf{W}_{u}$ and $\lambda$ presented in Table 1 . All the weights $\boldsymbol{\Sigma}_{\mathbf{e}}^{-1}(t)$, $t=0, \ldots, N-1$ have been scaled by $\boldsymbol{\Sigma}_{\mathbf{e}}^{-1}(0)$ to give values in the order of 1 to $10^{2}$ instead of values in the order of $10^{4}$ to $10^{6}$. Note that the scaling does not affect the mutual values of the weights.

The mean relative reduction of the RMSE over 100 Monte Carlo (MC) simulations is presented in Fig 3, where it can be seen how the parameters $\mathbf{W}_{\mathbf{u}}$ and $\lambda$ affect the error. The weight $\mathbf{W}_{\mathbf{u}}$ affect the final error, where a larger value gives a larger error. For $\lambda$ it can be seen that a larger value gives a slower convergence rate. The results are in accordance with the linear case, see e.g. [9].
Table 1

Parameters for the ILC update law used in the simulations.

\begin{tabular}{c|ccccc} 
Test & 1 & 2 & 3 & 4 & 5 \\
\hline $\mathbf{W}_{u}$ & 0.1 & 0.01 & 1 & 0.1 & 0.1 \\
$\lambda$ & 1 & 1 & 1 & 0.1 & 10
\end{tabular}

The simulation results show that the norm-optimal ILC using a linearised model around the estimated state trajectory from the previous ILC iteration works good for the flexible joint model. However, the tuning of $\mathbf{W}_{\mathbf{u}}$ and $\lambda$ is more difficult to deal with, than in the linear case. The difficulty comes from the fact that the choice of $\lambda$ can affect the error between the linearised model from previous iteration and the actual model. A low value of $\lambda$ can make the difference $\mathbf{u}_{k}-\mathbf{u}_{k-1}$ large, hence the linearised model can differ a lot from the actual.

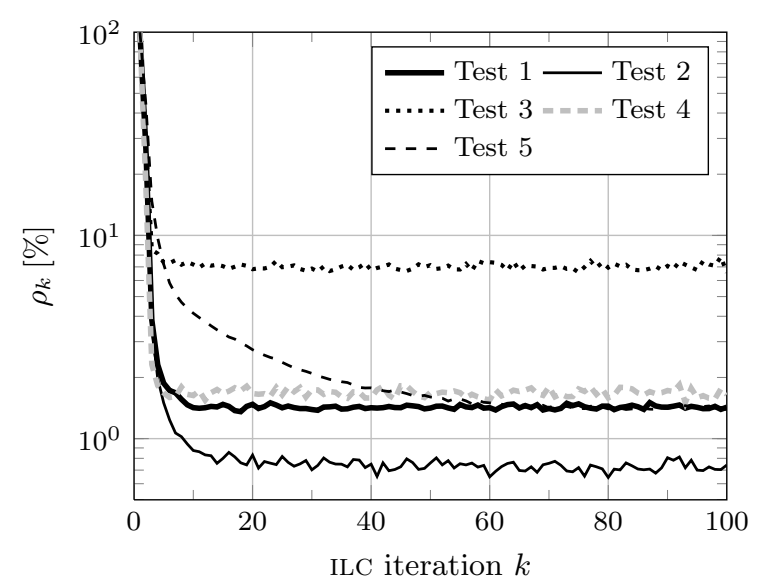

Fig. 3. Mean value of the relative reduction of the RMSE over 100 MC simulations.

\section{Conclusions}

An estimation-based norm-optimal ILC control law was derived together with stability and convergence results. The regular optimisation criteria for norm-optimal ILC was extended to an optimisation criteria including the first and second order moment of the posterior PDF. For LTI-systems it was shown that the control law can be separated from the estimator dynamics. Extensions to non-linear systems using linearisation was also presented. Simulations on a simplified non-linear model of an industrial manipulator show that the proposed ILC method works. The estimation-based norm-optimal ILC framework enables a systematic model-based design of ILC algorithms for non-linear systems.

\section{Acknowledgements}

This work was supported by the Vinnova Excellence Center LINK-SIC. 


\section{References}

[1] H.-S. Ahn, K. L. Moore, and Y. Chen. Kalman filteraugmented iterative learning control on the iteration domain. In Proceedings of the American Control Conference, pages 250-255, Minneapolis, MN, USA, June 2006.

[2] N. Amann, D. H. Owens, and E. Rogers. Iterative learning control for discrete-time systems with exponential rate of convergence. IEE Proceedings Control Theory and Applications, 143(2):217-224, March 1996.

[3] N. Amann, D. H. Owens, and E. Rogers. Iterative learning control using optimal feedback and feedforward actions. International Journal of Control, 65(2):277-293, 1996.

[4] B. D. O. Anderson and J. B. Moore. Optimal Filtering. Information and System Sciences Series. Prentice Hall Inc., Englewood Cliffs, NJ, USA, 1979.

[5] S. Arimoto, S. Kawamura, and F. Miyazaki. Bettering operation of robots by learning. Journal of Robotic Systems, 1(2):123-140, 1984.

[6] C. Arndt. Information Measures. Springer-Verlag, Berlin, Heidelberg, Germany, 2001.

[7] M. S. Arulampalam, S. Maskell, N. Gordon, and T. Clapp. A tutorial on particle filters for online nonlinear/non-Gaussian Bayesian tracking. IEEE Transactions on Signal Processing, 50(2):174-188, February 2002.

[8] P. Axelsson, R. Karlsson, and M. Norrlöf. Estimationbased ILC using particle filter with application to industrial manipulators. In Proceedings of the IEEE/RSJ International Conference on Intelligent Robots and Systems, Tokyo, Japan, November 2013. Accepted for publication.

[9] K. Barton, J. van de Wijdeven, A. Alleyne, O. Bosgra, and M. Steinbuch. Norm optimal cross-coupled iterative learning control. In Proceedings of the 47th IEEE Conference on Decision and Control, pages 3020-3025, Cancun, Mexico, December 2008.

[10] Z. Bien and J.-X. Xu. Iterative Learning Control: Analysis, Design, Integration and Application. Kluwer Academic Publishers, Boston, MA, USA, 1998.

[11] D. A. Bristow, M. Tharayil, and A. G. Alleyne. A survey of iterative learning control - a learning-based method for high-performance tracking control. IEEE Control Systems Magazine, 26:96-114, June 2006.

[12] A. Doucet, N. de Freitas, and N. Gordon, editors. Sequential Monte Carlo Methods in Practice. Statistics for Engineering and Information Science. Springer, New York, NY, USA, 2001.

[13] N. J. Gordon, D. J. Salmond, and A. F. M. Smith. Novel approach to nonlinear/non-Gaussian Bayesian state estimation. IEE Proceedings on Radar and Signal Processing, 140(2):107-113, April 1993.

[14] S. Gunnarsson and M. Norrlöf. On the design of ILC algorithms using optimization. Automatica, 37(12):20112016, 2001.

[15] A. H. Jazwinski. Stochastic Processes and Filtering Theory, volume 64 of Mathematics in Science and Engineering. Academic Press, New York, NY, USA, 1970.

[16] T. Kailath, A. H. Sayed, and B. Hassibi. Linear Estimation. Information and System Sciences Series. Prentice Hall Inc., Upper Saddle River, NJ, USA, 2000.

[17] R. E. Kalman. A new approach to linear filtering and prediction problems. Transactions of the AMSE-Journal of Basic Engineering, 82(Series D):35-45, 1960.
[18] S. Kullback and R. A. Leibler. On information and sufficiency. Annals of Mathematical Statistics, 22(1):79-86, March 1951.

[19] K. S. Lee and J. H. Lee. Iterative Learning Control: Analysis, Design, Integration and Application, chapter Design of Quadratic Criterion-based Iterative Learning Control, pages 165-192. Kluwer Academic Publishers, Boston, MA, USA, 2008.

[20] S. Moberg, J. Öhr, and S. Gunnarsson. A benchmark problem for robust control of a multivariable nonlinear flexible manipulator. In Proceedings of the 17th IFAC World Congress, pages 1206-1211, Seoul, Korea, July 2008.

[21] K. L. Moore. Iterative Learning Control for Deterministic Systems. Advances in Industrial Control. Springer-Verlag, London, UK, 1993.

[22] J. Nocedal and S. J. Wright. Numerical Optimization. Springer Series in Operations Research. Springer, New York, NY, USA, second edition, 2006.

[23] M. Norrlöf and S. Gunnarsson. Time and frequency domain convergence properties in iterative learning control. International Journal of Control, 75(14):1114-1126, 2002.

[24] W. J. Rugh. Linear System Theory. Information and System Sciences Series. Prentice Hall Inc., Upper Saddle River, NJ, USA, second edition, 1996.

[25] J. Wallén, I. Dressler, A. Robertsson, M. Norrlöf, and S. Gunnarsson. Observer-based ILC applied to the GantryTau parallel kinematic robot. In Proceedings of the 18th IFAC World Congress, pages 992-998, Milano, Italy, August/September 2011.

[26] J. Wallén, S. Gunnarsson, R. Henriksson, S. Moberg, and M. Norrlöf. ILC applied to a flexible two-link robot model using sensor-fusion-based estimates. In Proceedings of the 48th IEEE Conference on Decision and Control, pages 458463, Shanghai, China, December 2009.

[27] J. Wallén, M. Norrlöf, and S. Gunnarsson. A framework for analysis of observer-based ILC. Asian Journal of Control, 13(1):3-14, January 2011.

\section{A State Space Model and Kalman Filter in Batch Form}

For the norm-optimal ILC algorithm it is convenient to describe the state space model over the whole time horizon in batch form. The discrete-time state space model

$$
\begin{aligned}
\mathbf{x}(t+1) & =\mathbf{A}(t) \mathbf{x}(t)+\mathbf{B}_{\mathbf{u}}(t) \mathbf{u}(t) \\
\mathbf{y}(t) & =\mathbf{C}_{\mathbf{y}}(t) \mathbf{x}(t)+\mathbf{D}_{\mathbf{y u}}(t) \mathbf{u}(t)
\end{aligned}
$$

has the following update formula for the state vector [24]

$$
\mathbf{x}(t)=\phi\left(t, t_{0}\right) \mathbf{x}\left(t_{0}\right)+\sum_{j=t_{0}}^{t-1} \phi(t, j+1) \mathbf{B}_{\mathbf{u}}(j) \mathbf{u}(j)
$$

for $t \geq t_{0}+1$, where the discrete-time transition matrix $\phi(t, j)$ is defined by

$$
\phi(t, j)= \begin{cases}\mathbf{A}(t-1) \cdot \ldots \cdot \mathbf{A}(j), & t \geq j+1 \\ \mathbf{I}, & t=j\end{cases}
$$


Using (A.1b) and (A.2), the output is given by

$$
\begin{aligned}
\mathbf{y}(t)= & \mathbf{C}_{\mathbf{y}}(t) \boldsymbol{\phi}\left(t, t_{0}\right) \mathbf{x}\left(t_{0}\right)+\mathbf{D}_{\mathbf{y u}}(t) \mathbf{u}(t) \\
& +\sum_{j=t_{0}}^{t-1} \mathbf{C}_{\mathbf{y}}(t) \boldsymbol{\phi}(t, j+1) \mathbf{B}_{\mathbf{u}}(j) \mathbf{u}(j)
\end{aligned}
$$

Introducing the vectors

$$
\begin{aligned}
& \mathbf{x}=\left(\begin{array}{lll}
\mathbf{x}\left(t_{0}\right)^{\top} \ldots \mathbf{x}\left(t_{0}+N\right)^{\top} & \ldots
\end{array}\right)^{\top} \\
& \mathbf{u}=\left(\begin{array}{lll}
\mathbf{u}\left(t_{0}\right)^{\top} & \ldots & \mathbf{u}\left(t_{0}+N\right)^{\top}
\end{array}\right)^{\top} \\
& \mathbf{y}=\left(\begin{array}{lll}
\mathbf{y}\left(t_{0}\right)^{\top} & \ldots & \mathbf{y}\left(t_{0}+N\right)^{\top}
\end{array}\right)^{\top}
\end{aligned}
$$

gives the solution from $t=t_{0}$ to $t=t_{0}+N$ as $\mathbf{x}=$ $\boldsymbol{\Phi} \mathbf{x}\left(t_{0}\right)+\boldsymbol{\Psi} \mathcal{B}_{\mathbf{u}} \mathbf{u}$ and for the output as

$$
\mathbf{y}=\mathcal{C}_{\mathbf{y}} \boldsymbol{\Phi} \mathbf{x}\left(t_{0}\right)+\underbrace{\left(\mathcal{C}_{\mathbf{y}} \boldsymbol{\Psi} \mathcal{B}_{\mathbf{u}}+\mathcal{D}_{\mathbf{y u}}\right)}_{\triangleq \mathbf{T}_{\mathbf{y u}}} \mathbf{u} .
$$

Here $\mathbf{x}\left(t_{0}\right)$ is the initial value, and

$$
\begin{aligned}
& \mathcal{B}_{\mathbf{u}}=\operatorname{diag}\left(\mathbf{B}_{\mathbf{u}}\left(t_{0}\right), \ldots, \mathbf{B}_{\mathbf{u}}\left(t_{0}+N-1\right), 0\right) \\
& \mathcal{C}_{\mathbf{y}}=\operatorname{diag}\left(\mathbf{C}_{\mathbf{y}}\left(t_{0}\right), \ldots, \mathbf{C}_{\mathbf{y}}\left(t_{0}+N\right)\right) \\
& \mathcal{D}_{\mathbf{y u}}=\operatorname{diag}\left(\mathbf{D}_{\mathbf{y u}}\left(t_{0}\right), \ldots, \mathbf{D}_{\mathbf{y u}}\left(t_{0}+N\right)\right) \\
& \boldsymbol{\Phi}=\left(\begin{array}{c}
\mathbf{I} \\
\boldsymbol{\phi}\left(t_{0}+1, t_{0}\right) \\
\boldsymbol{\phi}\left(t_{0}+2, t_{0}\right) \\
\vdots \\
\boldsymbol{\phi}\left(t_{0}+N, t_{0}\right)
\end{array}\right) \\
& \boldsymbol{\Psi}=\left(\begin{array}{ccccc}
0 & 0 & 0 & \cdots & 0 \\
\mathbf{I} & 0 & 0 & \cdots & 0 \\
\boldsymbol{\phi}\left(t_{0}+2, t_{0}+1\right) & \mathbf{I} & 0 & \cdots & 0 \\
\vdots & \vdots & \ddots & \ddots & \vdots \\
\boldsymbol{\phi}\left(t_{0}+N, t_{0}+1\right) & \boldsymbol{\phi}\left(t_{0}+N, t_{0}+2\right) & \cdots & \mathbf{I} & 0
\end{array}\right)
\end{aligned}
$$

\section{A.1 Kalman Filter}

The KF can be written in a similar batch form as described above. Let the state space model be given by

$$
\begin{aligned}
\mathbf{x}(t+1) & =\mathbf{A}(t) \mathbf{x}(t)+\mathbf{B}_{\mathbf{u}}(t) \mathbf{u}(t)+\mathbf{G}(t) \mathbf{w}(t) \\
\mathbf{y}(t) & =\mathbf{C}_{\mathbf{y}}(t) \mathbf{x}(t)+\mathbf{D}_{\mathbf{y u}}(t) \mathbf{u}(t)+\mathbf{v}(t)
\end{aligned}
$$

where $\mathbf{w}(t) \sim \mathcal{N}(\mathbf{0}, \mathbf{Q}(t))$, and $\mathbf{v}(t) \sim \mathcal{N}(\mathbf{0}, \mathbf{R}(t))$. From the KF recursions we get

$$
\begin{aligned}
& \hat{\mathbf{x}}(t+1 \mid t+1)=\left(\mathbf{I}-\mathbf{K}(t+1) \mathbf{C}_{\mathbf{y}}(t+1)\right) \mathbf{A}(t) \hat{\mathbf{x}}(t \mid t) \\
& +\left(\mathbf{I}-\mathbf{K}(t+1) \mathbf{C}_{\mathbf{y}}(t+1)\right) \mathbf{B}_{\mathbf{u}}(t) \mathbf{u}(t) \\
& -\mathbf{K}(t+1) \mathbf{D}_{\mathbf{y u}}(t+1) \mathbf{u}(t+1)+\mathbf{K}(t+1) \mathbf{y}(t+1) \\
& =\widetilde{\mathbf{A}}(t) \hat{\mathbf{x}}(t \mid t)+\widetilde{\mathbf{B}}_{\mathbf{y u}}(t) \mathbf{u}(t)-\widetilde{\mathbf{D}}_{\mathbf{y u}}(t+1) \mathbf{u}(t+1) \\
& +\mathbf{K}(t+1) \mathbf{y}(t+1)
\end{aligned}
$$

where, $\mathbf{K}(t)$ is the Kalman gain which can be computed using the recursion

$$
\begin{aligned}
\mathbf{P}(t \mid t-1)= & \mathbf{A}(t-1) \mathbf{P}(t-1 \mid t-1) \mathbf{A}(t-1)^{\top} \\
& +\mathbf{G}(t-1) \mathbf{Q}(t-1) \mathbf{G}(t-1)^{\top} \\
\mathbf{K}(t)= & \mathbf{P}(t \mid t-1) \mathbf{C}_{\mathbf{y}}(t)^{\top} \\
& \times\left(\mathbf{C}_{\mathbf{y}}(t) \mathbf{P}(t \mid t-1) \mathbf{C}_{\mathbf{y}}(t)^{\top}+\mathbf{R}(t)\right)^{-1} \\
\mathbf{P}(t \mid t)= & \left(\mathbf{I}-\mathbf{K}(t) \mathbf{C}_{\mathbf{y}}(t)\right) \mathbf{P}(t \mid t-1) .
\end{aligned}
$$

The update formula for the estimated state vector can now be written as

$$
\begin{gathered}
\hat{\mathbf{x}}(t \mid t)=\widetilde{\phi}\left(t, t_{0}\right) \hat{\mathbf{x}}\left(t_{0} \mid t_{0}\right)+\sum_{j=t_{0}}^{t-1} \widetilde{\phi}(t, j+1) \widetilde{\mathbf{B}}_{\mathbf{y u}}(j) \mathbf{u}(j) \\
-\sum_{j=t_{0}+1}^{t} \widetilde{\phi}(t, j) \widetilde{\mathbf{D}}_{\mathbf{y u}}(j) \mathbf{u}(j)+\sum_{j=t 0+1}^{t} \widetilde{\phi}(t, j) \mathbf{K}(j) \mathbf{y}(j),
\end{gathered}
$$

where $\widetilde{\boldsymbol{\phi}}$ is defined as in (A.3), with $\widetilde{\mathbf{A}}(t)$ instead of $\mathbf{A}(t)$.

The KF recursion in batch form becomes

$$
\hat{\mathbf{x}}=\widetilde{\boldsymbol{\Phi}} \hat{\mathbf{x}}\left(t_{0} \mid t_{0}\right)+\left(\widetilde{\mathbf{\Psi}} \widetilde{\mathcal{B}}_{\mathbf{y u}}-\widetilde{\boldsymbol{\Psi}}_{2} \widetilde{\mathcal{D}}_{\mathbf{y u}}\right) \mathbf{u}+\widetilde{\boldsymbol{\Psi}}_{2} \mathcal{K} \mathbf{y}
$$

where $\widetilde{\boldsymbol{\Phi}}, \widetilde{\boldsymbol{\Psi}}$, and $\widetilde{\mathcal{B}}_{\text {yu }}$ are given in (A.9) with $\widetilde{\mathbf{A}}(t)$ and $\widetilde{\mathbf{B}}_{\mathbf{y u}}(t)$ instead of $\mathbf{A}(t)$ and $\mathbf{B}(t)$. The remaining matrices are defined as

$$
\begin{aligned}
\widetilde{\boldsymbol{\Psi}}_{2} & =\left(\mathbf{0}_{(N+1) n_{x} \times n_{x}} \widetilde{\boldsymbol{\Psi}}\left(\begin{array}{c}
\mathbf{I}_{N n_{x}} \\
\mathbf{0}_{n_{x} \times N n_{x}}
\end{array}\right)\right) \\
\widetilde{\mathcal{D}}_{\mathbf{y u}} & =\operatorname{diag}\left(\mathbf{0}, \widetilde{\mathbf{D}}_{\mathbf{y u}}\left(t_{0}+1\right), \ldots, \widetilde{\mathbf{D}}_{\mathbf{y u}}\left(t_{0}+N\right)\right) \\
\mathcal{K} & =\operatorname{diag}\left(\mathbf{0}, \mathbf{K}\left(t_{0}+1\right), \ldots, \mathbf{K}\left(t_{0}+N\right)\right) .
\end{aligned}
$$

Assuming that not the state vector is of interest, but instead a variable given by

$$
\mathbf{z}(t)=\mathbf{C}_{\mathbf{z}}(t) \mathbf{x}(t)+\mathbf{D}_{\mathbf{z u}}(t) \mathbf{u}(t)
$$


gives the batch formulation

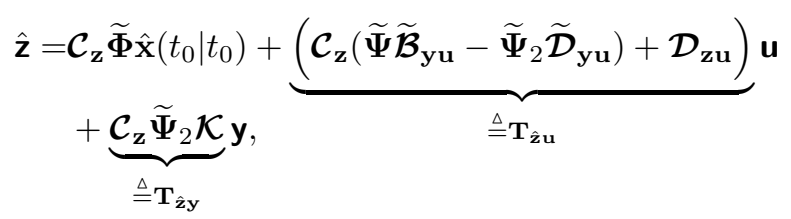

where $\mathcal{C}_{\mathbf{z}}$ and $\mathcal{D}_{\mathbf{z u}}$ are given in (A.9) using $\mathbf{C}_{\mathbf{z}}(t)$ and $\mathbf{D}_{\mathbf{z u}}(t)$. 


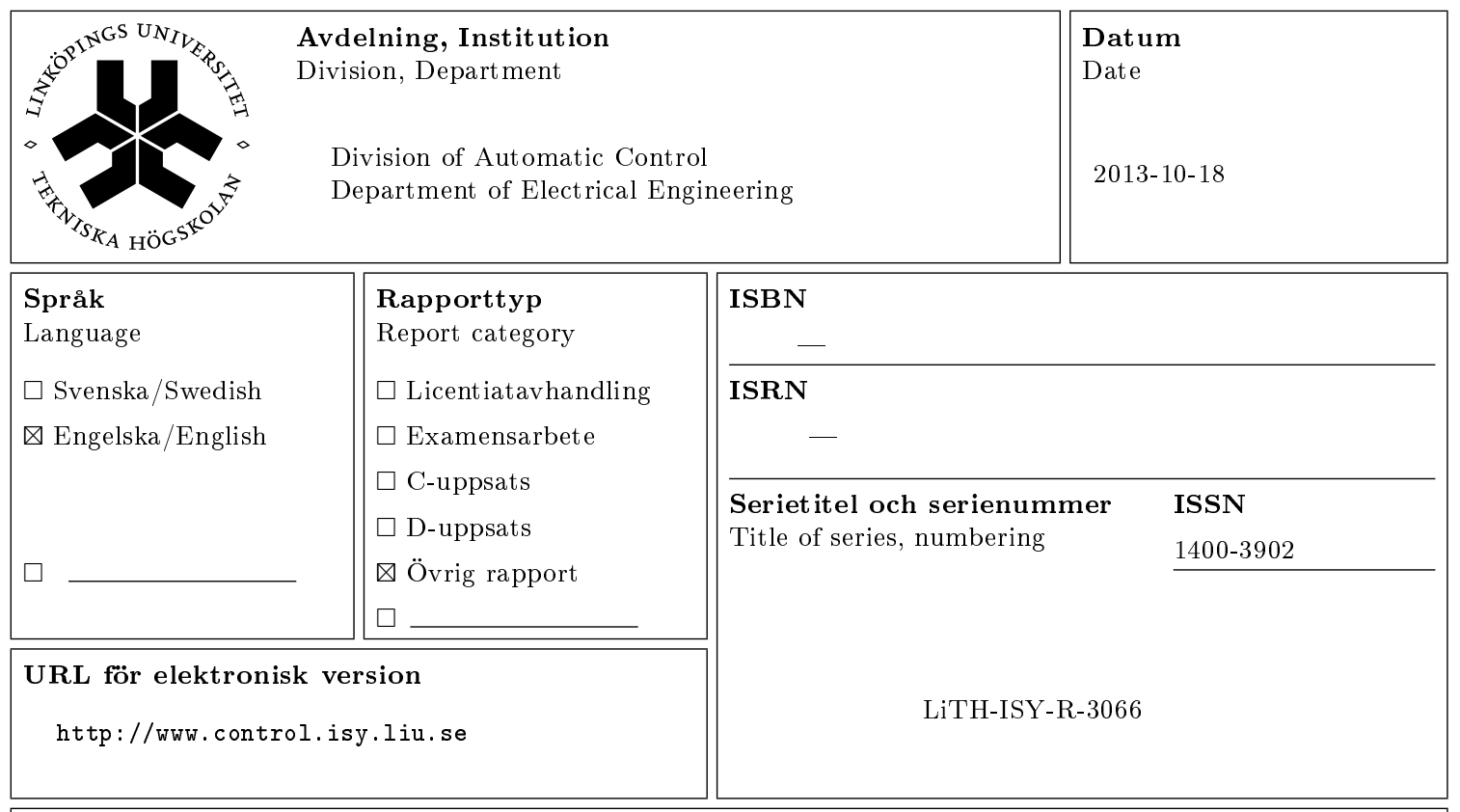

Titel Estimation-based Norm-optimal Iterative Learning Control

Title

Estimation-based Norm-optimal Iterative Learning Control

Författare Patrik Axelsson, Rickard Karlsson, Mikael Norrlöf

Author

Sammanfattning

Abstract

The iterative learning control (ILC) method improves performance of systems that repeat the same task several times. In this paper the standard norm-optimal ILC control law for linear systems is extended to an estimation-based ILC algorithm where the controlled variables are not directly available as measurements. The proposed ILC algorithm is proven to be stable and gives monotonic convergence of the error. The estimation-based part of the algorithm uses Bayesian estimation techniques such as the Kalman filter. The objective function in the optimisation problem is modified to incorporate not only the mean value of the estimated variable, but also information about the uncertainty of the estimate. It is further shown that for linear time-invariant systems the ILC design is independent of the estimation method. Finally, the concept is extended to non-linear state space models using linearisation techniques, where it is assumed that the full state vector is estimated and used in the ILC algorithm. It is also discussed how the Kullback-Leibler divergence can be used if linearisation cannot be performed. Finally, the proposed solution for non-linear systems is applied and verified in a simulation study with a simplified model of an industrial manipulator system. 